\title{
A Comparison of Weekly DCF versus Standard DCF as First-line Systemic Chemotherapy for Metastatic Gastric Cancer Patients
}

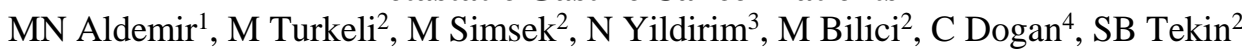

\begin{abstract}
Objective: In metastatic gastric cancer (MGC), the goals of treatment are palliation and prolongation of overall survival (OS). Systemic chemotherapy, with docetaxel, cisplatin, infusional 5-fluorouracil (DCF) the most preferred regimen, is the mainstay of the treatment. We evaluated the efficacy and tolerability of weekly DCF (wDCF) compared to standard DCF regimen.
\end{abstract}

Methods: We retrospectively reviewed 49 and 32 MGC patients treated with DCF and wDCF regimens as first-line treatment, respectively. The wDCF protocol included $25 \mathrm{mg} / \mathrm{m}^{2}$ docetaxel, $25 \mathrm{mg} / \mathrm{m}^{2}$ cisplatin and $750 \mathrm{mg} / \mathrm{m}^{2}$ infusional 5 -FU on day 1 , every week. Each cycle was repeated every 3 weeks.

Results: The patients in wDCF arm were significantly older (median 54 vs 72.5) and had poor Eastern Cooperative Oncology Group performance status (ECOG PS) than patients in DCF arm $(\mathrm{p}<0.001)$. PFS was 3 vs 5 months ( $>0.05)$ and OS was 7 vs 9 months in DCF and wDCF arms (p>0.05), respectively. Overall response rate (ORR) was observed in $28.5 \%$ and $31.2 \%$ of the patients in DCF and wDCF arms, respectively $(\mathrm{p}=0.65)$. Hematologic toxicities were observed more common in wDCF arm.

Conclusions: wDCF is an effective and tolerable regimen and may be an alternative in patients who are elderly and have poor ECOG PS.

Keywords: Cisplatin, docetaxel, infusional 5-fluorouracil, metastatic gastric cancer

From: ${ }^{1}$ Erzincan University Medicine Faculty Medical Oncology Department, Erzincan, Turkey, ${ }^{2}$ Ataturk University Medicine Faculty Medical Oncology Department, Erzurum, Turkey, ${ }^{3}$ Dr. Ersin Arslan Training and Research Hospital, Medical Oncology Department, Gaziantep, Turkey, ${ }^{4}$ Ataturk University Medicine Faculty Internal Medicine Department, Erzurum, Turkey.

Correspondence: Dr MN Aldemir,Department of Medical Oncology, Faculty of Medicine, Erzincan University, Erzincan, Turkey. Fax: +90 44621222 11, e-mail: aldemirmn@ gmail.com 


\section{INTRODUCTION}

Gastric cancer is a common type of cancer worldwide and represents an important cause of cancer-related mortality. Gastric cancer is more frequently seen in men than women, in both developed and developing countries (1).

About two third of gastric cancer patients are in metastatic stage at diagnosis. While metastatic gastric cancer (MGC) is a noncurable disease, the goals of treatment are palliation and prolongation of overall survival (OS). Systemic chemotherapy is the most effective treatment modality for these patients (2). In a meta-analysis of three trials comparing chemotherapy results versus best supportive care (BSC) in MGC patients, a significant benefit in OS in favor of chemotherapy was reported (hazard ratio [HR] 0.37, 95\% CI 0.24-0.55), which translated into an improvement in median OS from 4.3 to 11 months (3).

Despite large number of randomized trials, there is no consensus on the best chemotherapy regimen for initial chemotherapy of MGC. Although response rates are higher with combination regimens than single agents, only a modest longer duration of disease control and OS can be achieved which measured in weeks to a few months. The ECF (epirubicin, cisplatin, infusional 5-fluorouracil (5-FU)) and DCF (docetaxel, cisplatin, infusional 5-FU) combinations have emerged as standard regimens for first-line treatment in randomized trials $(4,5)$. In a randomized phase-II trial involving 81 patients, DCF was directly compared with ECF in first-line advanced gastric cancer and a trend favoring DCF over ECF was reported (6). In a phase III study comparing DCF versus CF (cisplatin, infusional 5-FU), DCF was reported to be significantly better (7). But in both trials the incidence of grade 3 or 4 diarrhea and neutropenia was higher with DCF.

Because of higher toxicity rates reported for DCF, several modifications of standard schedule have been investigated. It was aimed to alter the dose and frequency of the cytotoxic 
agents to allow less toxicity with no worsening on efficacy. With these affords, modified schedules for DCF were shown to have preserved efficacy and improved tolerability $(8,9)$. Weekly DCF (wDCF) is one of the alternatives of these affords. In a retrospective study, efficacy and tolerability of wDCF in advanced gastric and esophageal cancer patients not candidates for standard DCF was evaluated. It was reported that wDCF demonstrated a modest activity with minimal hematologic toxicity, suggesting that wDCF may be a treatment option in these patients (10).

We performed a retrospective analysis of the efficacy and tolerability of wDCF versus DCF in MGC patients in the first-line treatment.

\section{PATIENTS AND METHODS}

We retrospectively reviewed 81 MGC patients who were treated with DCF or wDCF as firstline treatment from April 2007 to July 2014 in Ataturk University, School of Medicine, Department of Medical Oncology.

The DCF protocol included $75 \mathrm{mg} / \mathrm{m}^{2}$ docetaxel, $75 \mathrm{mg} / \mathrm{m}^{2}$ cisplatin on day 1 and 750 $\mathrm{mg} / \mathrm{m}^{2} /$ daily, days 1 to 5 infusional 5-FU, repeated every 3 weeks. Primary prophylaxis with white blood cell growth factor support was used in patient who received DCF regimen. The wDCF protocol included $25 \mathrm{mg} / \mathrm{m}^{2}$ docetaxel, $25 \mathrm{mg} / \mathrm{m}^{2}$ cisplatin and $750 \mathrm{mg} / \mathrm{m}^{2}$ infusional 5-FU on day 1, every week. Each cycle was repeated every 3 weeks.

Study inclusion criteria were MGC diagnosed as adenocarcinoma at referral, Eastern Cooperative Oncology Group (ECOG) performance status (PS) to be 0,1 or 2 at diagnosis, receiving at least two cycles of DCF or wDCF as first-line chemotherapy. Patients undergone previous curative operation and received adjuvant treatment were excluded.

Disease and patient characteristics, prognostic factors, treatment response, grade 3-4 hematologic and non-hematologic treatment toxicities, progression free survival (PFS) and 
OS were evaluated. Grade 3-4 toxicities were assessed according to National Cancer Institute Common Toxicity Criteria (NCICTC) (version 4.0). Imaging studies were documented by computed tomography and positron emission tomography-computed tomography (PET-CT) at baseline for every three cycles. Tumor responses were determined using Response Evaluation Criteria in Solid Tumors (RECIST) (version 1.1) (11).

\section{Statistical analysis}

Analyses were performed using the SPSS statistical software program package (SPSS version 20.0 for windows). Survival curves were established with The Kaplan-Meier method. PFS was defined as the time from MGC diagnosis to disease progression or death. OS was defined as the time from MGC diagnosis to death from any cause. PFS and OS were calculated with the log-rank test. The differences of the clinical characteristics between two groups were analyzed by chi-square test and Mann Whitney U test. Differences were assumed to be significant when $\mathrm{p}$ value was less than 0.05 .

\section{RESULTS}

\section{Patient characteristics}

From total of 81 enrolled patients, 49 and 32 patients were treated with DCF and wDCF regimens, respectively. Demographical and clinical characteristics of patients were listed in Table 1.

The patients in wDCF arm were significantly older than patients in DCF arm (median 54 vs $72.5, \mathrm{p}<0.001)$. While the ratio of patients over 65 years old in DCF arm was $12.2 \%$, it was $84.4 \%$ in wDCF arm. The patients in wDCF arm had significantly poor ECOG PS than DCF arm $(p<0.001)$. The ratio of ECOG PS 2 patients were $59.4 \%$ in wDCF arm whereas 
$10.2 \%$ in DCF arm. The median follow-up time was 9 (1-60) months and was not significant between two groups $(\mathrm{p}=0.65)$.

\section{Efficacy}

In DCF arm patients were received median 6 (2-8) cycles of chemotherapy, whereas in wDCF arm median 3.5 (2-8) cycles. PFS was 3 (95\%CI: 0.6-5.4) months in wDCF arm and 5 (95\%CI: 4.1-5.9) months in DCF arm (p=0.75). OS was 7 (95\%CI: 5.6-8.4) months in wDCF arm and 9 (95\%CI: 6.9-11) months in DCF arm ( $\mathrm{p}=0.33)$. Although, both PFS and OS were shorter in wDCF arm, there was no statistically significant difference between two arms (Figure 1). Overall response rate (ORR) was observed in $28.5 \%$ and $31.2 \%$ of the patients in DCF and wDCF arms, respectively $(\mathrm{p}=0.65)$. The treatment efficacy and ORR are summarized in Table 2.

\section{Safety and tolerability}

Dose reduction was required in $12(24.5 \%)$ patients in DCF arm and $4(12.5 \%)$ patients in wDCF $\operatorname{arm}(\mathrm{p}=0.19)$. Dose was delayed in $12(24.5 \%)$ patients in DCF arm, whereas in 10 (31.3\%) patients in wDCF arm $(\mathrm{p}=0.5)$. The hematologic and non-hematologic toxicities were summarized in Table 3. Anemia, neutropenia and thrombocytopenia were observed more frequently in wDCF arm. No patients died due to chemotherapy toxicity.

\section{DISCUSSION}

Gastric cancer is a common form of cancer worldwide. Gastric cancer is a highly mortal malignancy. About two third of gastric cancer patients are in metastatic stage at diagnosis. The five-year OS for gastric cancer is lower than most of other cancer forms (1). Better OS was demonstrated with chemotherapy compared to BSC in MGC patients. But the optimal treatment regimen is not clear yet for initial chemotherapy in this population. 
Combination regimens seem to be more effective than single agent treatments (3). DCF is a commonly used regimen in MGC as first-line chemotherapy with a low tolerability rate due to its toxicity. The largest phase 3 randomized study comparing DCF to cisplatin plus 5-FU demonstrated superiority of triplet chemotherapy. ORR (37\% vs $25 \%)$, median time to progression (TTP) (5.6 vs 3.7 months), and median OS (9.2 vs 8.6 months) was significantly better with DCF than CF (5). In a retrospective study comparing DCF to modified DCF $(\mathrm{mDCF})$, response rates were similar in both arms. There was no statistically significant difference in terms of complete response (CR) (10.3\% vs 6.7\%), partial response (PR) $(35.3 \%$ vs $40.0 \%$ ), stable disease (SD) (32.4\% vs $33.3 \%$ ), progressive disease (PD) (22.1\% vs $20.0 \%$ ) and ORR (45.6\% vs 46.7\%). PFS was more favorable in DCF arm than mDCF arm, but the difference was not statistically significant ( 7.4 vs 6.5 months, OS was not significantly superior in DCF arm (9.9 vs 8.6 months (9). In another study comparing DCF to mDCF, comparable efficacy was reported with $\mathrm{mDCF}$ and $\mathrm{CR}$ was $2 \%$ vs $5.4 \%$, PR was $35 \%$ vs $21.6 \%$, SD was $30 \%$ vs $37.9 \%$, PD was $17 \%$ vs $13.5 \%$ and ORR was $37 \%$ vs $27 \%$, respectively (12).

Overman et al. retrospectively evaluated the efficacy and tolerability of wDCF in patients with advanced gastric and esophageal cancer who were not candidates for DCF. In wDCF arm, treatment was administered as $20 \mathrm{mg} / \mathrm{m}^{2}$ of docetaxel, $20 \mathrm{mg} / \mathrm{m}^{2}$ of cisplatin, and $350 \mathrm{mg} / \mathrm{m}^{2}$ of 5 -FU once weekly for 6 consecutive weeks followed by a 2 -week break. ORR was reported as 34\% (95\% CI, 24-45\%), PFS as 4.1 months (95\% CI, 3.6-5.7 months) and OS as 8.9 months (95\% CI, 7.7-10.8 months). The authors suggested that wDCF demonstrated modest activity with minimal hematologic toxicity and wDCF might be a reasonable treatment option for such patients (10).

In our study, PFS was 3 vs 5 months and OS was 7 vs 9 months in DCF and wDCF arms, respectively. Although, both PFS and OS were shorter in wDCF arm, there was no 
statistically significant difference between two arms. ORR was observed in $28.5 \%$ and $31.2 \%$ of the patients in DCF and wDCF arms, respectively.

In the V325 study significant toxicity was reported with DCF despite good ECOG PS (0-1) and young age of the patients (median age 55 years). Grade 3-4 toxicities were reported as neutropenia (82\%), anemia (18\%), thrombocytopenia (8\%), febrile neutropenia (29\%), nausea (14\%), vomiting (17\%), and diarrhea (19\%) (5). In another study designed retrospectively and compared DCF vs mDCF, grade 3-4 toxicities with DCF were determined as neutropenia (48.2\%), febrile neutropenia (19\%), thrombocytopenia (25.9\%), nausea (44.7\%) and vomiting $(31.8 \%)$ while toxicity rates with $\mathrm{mDCF}$ were neutropenia $(13.6 \%)$, febrile neutropenia (4.5\%), thrombocytopenia (9.1\%), nausea (13.6\%), and vomiting (4.5\%). Grade 3-4 toxicities were significantly less common in mDCF arm compared to DCF arm (9). Nuriye et al. reported grade 3-4 toxicities as neutropenia ( $82 \%$ vs $8.1 \%$ ), anemia (18\% vs 5.4\%), thrombocytopenia (17\% vs $0 \%$ ), nausea (14\% vs $5.4 \%)$, and vomiting (14\% vs $0 \%$ ) in their study comparing DCF vs mDCF, respectively (12). Overman et al reported grade 3-4 toxicities with wDCF as neutropenia (4\%), neutropenic fever $(0 \%)$, thrombocytopenia $(0 \%)$, and anemia (9\%) (10).

In our study grade $3-4$ toxicity rates were as neutropenia (4.1\% vs $15.6 \%)$, anemia (4.1\% vs $9.4 \%)$, thrombocytopenia (0\% vs $6.3 \%)$, and nausea-vomiting (6.1\% vs $3.1 \%$ ) in DCF arm compared to wDCF arm, respectively. Hematologic toxicities were observed more common in wDCF arm in our study. This may be due to higher rates of patients with older age and poor ECOG PS in wDCF arm as $84.4 \%$ were 65 years and older and $59.4 \%$ had ECOG PS 2 of our patients in wDCF arm.

Limitations of our study are its retrospective design, single center experience, and low number of patients included. 


\section{CONCLUSION}

In MGC, DCF is an effective and more tolerable regimen in young patients and patients with good ECOG PS. In our study although PFS and OS were shorter with wDCF compared to DCF, there was no statistically significant difference between two arms. We found that wDCF was an effective and tolerable regimen and may be an alternative in patients who are elderly and have poor ECOG PS. This subject should be investigated in large-numbered prospective randomized trials.

\section{AUTHORS' NOTE}

Conflict of interest: All authors declare that they have no conflicts of interest.

Financial Disclosure: This research received no specific grant from any funding agency in the public, commercial, or not-for-profit sectors.

Informed Consent: Because of the retrospective design of the study, no informed consent was obtained from the patients. Only ethics committee approval was obtained at the beginning of the study at number $4 / 31$ on date 28.04 .2016 


\section{REFERENCES}

1. Jemal A, Bray F, Center MM, Ferlay J, Ward E, Forman D. Global cancer statistics. CA: a cancer journal for clinicians. 2011; 61: 69-90.

2. Kamangar F, Dores GM, Anderson WF. Patterns of cancer incidence, mortality, and prevalence across five continents: defining priorities to reduce cancer disparities in different geographic regions of the world. Journal of clinical oncology. 2006; 24: 2137-50.

3. Wagner AD, Unverzagt S, Grothe W, Kleber G, Grothey A, Haerting J et al. Chemotherapy for advanced gastric cancer. Cochrane Database Syst Rev. 2010; 3: CD004064.

4. Webb A, Cunningham D, Scarffe JH, Harper P, Norman A, Joffe JK et al. Randomized trial comparing epirubicin, cisplatin, and fluorouracil versus fluorouracil, doxorubicin, and methotrexate in advanced esophagogastric cancer. J Clinic Oncol 1997; 15: 261-7.

5. Van Cutsem E, Moiseyenko VM, Tjulandin S, Majlis A, Constenla M, Boni C et al. Phase III study of docetaxel and cisplatin plus fluorouracil compared with cisplatin and fluorouracil as first-line therapy for advanced gastric cancer: a report of the V325 Study Group. J Clinic Oncol 2006; 24: 4991-7.

6. Roth AD, Fazio N, Stupp R, Falk S, Bernhard J, Saletti P et al. Docetaxel, cisplatin, and fluorouracil; docetaxel and cisplatin; and epirubicin, cisplatin, and fluorouracil as systemic treatment for advanced gastric carcinoma: a randomized phase II trial of the Swiss Group for Clinical Cancer Research. J Clinic Oncol 2007; 25: 3217-23. 
7. Ajani JA, Moiseyenko VM, Tjulandin S, Majlis A, Constenla M, Boni C et al. Clinical benefit with docetaxel plus fluorouracil and cisplatin compared with cisplatin and fluorouracil in a phase III trial of advanced gastric or gastroesophageal adenocarcinoma: the V-325 Study Group. J Clinic Oncol 2007; 25: 3205-9.

8. Shah MA, Jhawer M, Ilson DH, Lefkowitz RA, Robinson E, Capanu M et al. Phase II study of modified docetaxel, cisplatin, and fluorouracil with bevacizumab in patients with metastatic gastroesophageal adenocarcinoma. J Clinic Oncol 2011; 29: 868-74.

9. Inal A, Kaplan M, Kucukoner M, Isikdogan A. Docetaxel and Cisplatin Plus Fluorouracil compared with Modified Docetaxel, Cisplatin, and 5-Fluorouracil as firstline therapy for advanced gastric cancer: a retrospective analysis of single institution. Neoplasma. 2011; 59: 233-6.

10. Overman MJ, Kazmi SM, Jhamb J, Lin E, Yao JC, Abbruzzese JL et al. Weekly docetaxel, cisplatin, and 5-fluorouracil as initial therapy for patients with advanced gastric and esophageal cancer. Cancer 2010; 116: 1446-53.

11. Eisenhauer EA, Therasse P, Bogaerts J, Schwartz LH, Sargent D, Ford R et al. New response evaluation criteria in solid tumours: revised RECIST guideline (version 1.1). Euro J Cancer 2009; 45: 228-47.

12. Ozdemir NY, Abali H, Oksüzoğlu B, Budakoglu B, Uncu D, Güler T et al. The efficacy and safety of reduced-dose docetaxel, cisplatin, and 5-fluorouracil in the firstline treatment of advanced stage gastric adenocarcinoma. Med Oncol. 2010; 27: 6804. 
Table 1. Demographical and clinical characteristics of patients

\begin{tabular}{|c|c|c|c|c|c|}
\hline & \multicolumn{2}{|c|}{ DCF $(n=49)$} & \multicolumn{2}{|c|}{ wDCF $(n=32)$} & \multirow[b]{2}{*}{$p$} \\
\hline & $\mathrm{n}$ & $\%$ & $\mathrm{n}$ & $\%$ & \\
\hline \multicolumn{6}{|l|}{ Gender } \\
\hline Men & 34 & $\begin{array}{c}69 . \\
4\end{array}$ & 24 & 75 & 0.58 \\
\hline Women & 15 & $\begin{array}{c}30 . \\
6\end{array}$ & 8 & 25 & \\
\hline \multicolumn{6}{|l|}{ ECOG } \\
\hline 0 & 4 & 8.2 & 2 & 6.3 & $<0.001$ \\
\hline 1 & 40 & $\begin{array}{c}81 . \\
6\end{array}$ & 11 & 34.4 & \\
\hline 2 & 5 & $\begin{array}{c}10 . \\
2\end{array}$ & 19 & 59.4 & \\
\hline \multicolumn{6}{|l|}{ Age (years) } \\
\hline$<55$ & 26 & $\begin{array}{c}53 . \\
1\end{array}$ & 2 & 6.3 & $<0.001$ \\
\hline $55-64$ & 22 & $\begin{array}{c}44 . \\
9\end{array}$ & 3 & 9.4 & \\
\hline$\geq 65$ & 1 & 2 & 27 & 84.4 & \\
\hline \multicolumn{6}{|l|}{ Metastatic site } \\
\hline Liver & 37 & $\begin{array}{c}75 . \\
5\end{array}$ & 19 & 59.4 & 0.12 \\
\hline Lung & 2 & 4.1 & 7 & 21.9 & 0.01 \\
\hline Bone & 2 & 4.1 & 3 & 9.4 & 0.33 \\
\hline Peritoneal & 10 & $\begin{array}{c}20 . \\
4\end{array}$ & 11 & 34.4 & 0.16 \\
\hline \multicolumn{6}{|c|}{ Chemotherapy cycles } \\
\hline $2-3$ & 7 & $\begin{array}{c}14 . \\
3\end{array}$ & 16 & 50 & 0.002 \\
\hline $4-6$ & 40 & $\begin{array}{c}81 . \\
6\end{array}$ & 15 & 46.9 & \\
\hline$>6$ & 2 & 4.1 & 1 & 3.1 & \\
\hline \multicolumn{6}{|c|}{$\begin{array}{l}\text { Second line } \\
\text { chemotherapy }\end{array}$} \\
\hline Yes & 28 & $\begin{array}{c}57 . \\
1\end{array}$ & 9 & 28.1 & 0.01 \\
\hline No & 21 & $\begin{array}{c}42 . \\
9\end{array}$ & 23 & 71.9 & \\
\hline \multicolumn{6}{|l|}{ Last status } \\
\hline Dead & 44 & $\begin{array}{c}89 . \\
8\end{array}$ & 32 & 100 & \\
\hline Alive & 5 & $\begin{array}{c}10 . \\
2\end{array}$ & 0 & & \\
\hline
\end{tabular}


Table 2: Treatment efficacy of the patients

\begin{tabular}{|c|c|c|c|c|c|}
\hline & \multicolumn{2}{|c|}{ DCF $(n=49)$} & \multicolumn{2}{|c|}{ wDCF $(n=32)$} & \multirow[b]{2}{*}{$p$} \\
\hline & $\mathbf{n}$ & $\%$ & n & $\%$ & \\
\hline $\begin{array}{l}\text { Complete } \\
\text { response }\end{array}$ & 1 & 2 & 1 & 3.1 & 0.95 \\
\hline Partial response & 13 & 26.5 & 9 & 28.1 & \\
\hline Stable disease & 2 & 4.1 & 2 & 6.3 & \\
\hline $\begin{array}{l}\text { Progressive } \\
\text { disease }\end{array}$ & 33 & 67.3 & 20 & 62.5 & \\
\hline
\end{tabular}

Table 3: Grade 3-4 hematologic and non-hematologic toxicities (National Cancer Institute Common Toxicity Criteria, Version 4.0)

\begin{tabular}{cll}
\hline & \multicolumn{2}{l}{ No of patients (\%) } \\
& DCF & wDCF \\
\hline Hematologic toxicities & $2(4.1)$ & $5(15.6)$ \\
Neutropenia & $2(4.1)$ & $3(9.4)$ \\
Anemia & - & $2(6.3)$ \\
Thrombocytopenia & & \\
Non-hematologic toxicities & $5(10.2)$ & - \\
Diarrhea & $3(6.1)$ & $1(3.1)$ \\
Nausea-vomiting & $4(8.2)$ & - \\
Thrombosis & $1(2)$ & - \\
Mucositis & $1(2)$ & - \\
Neuropathy & $1(2)$ & $1(3.1)$ \\
Nephrotoxicity & $2(4.1)$ & $1(3.1)$ \\
Hepatotoxicity &
\end{tabular}



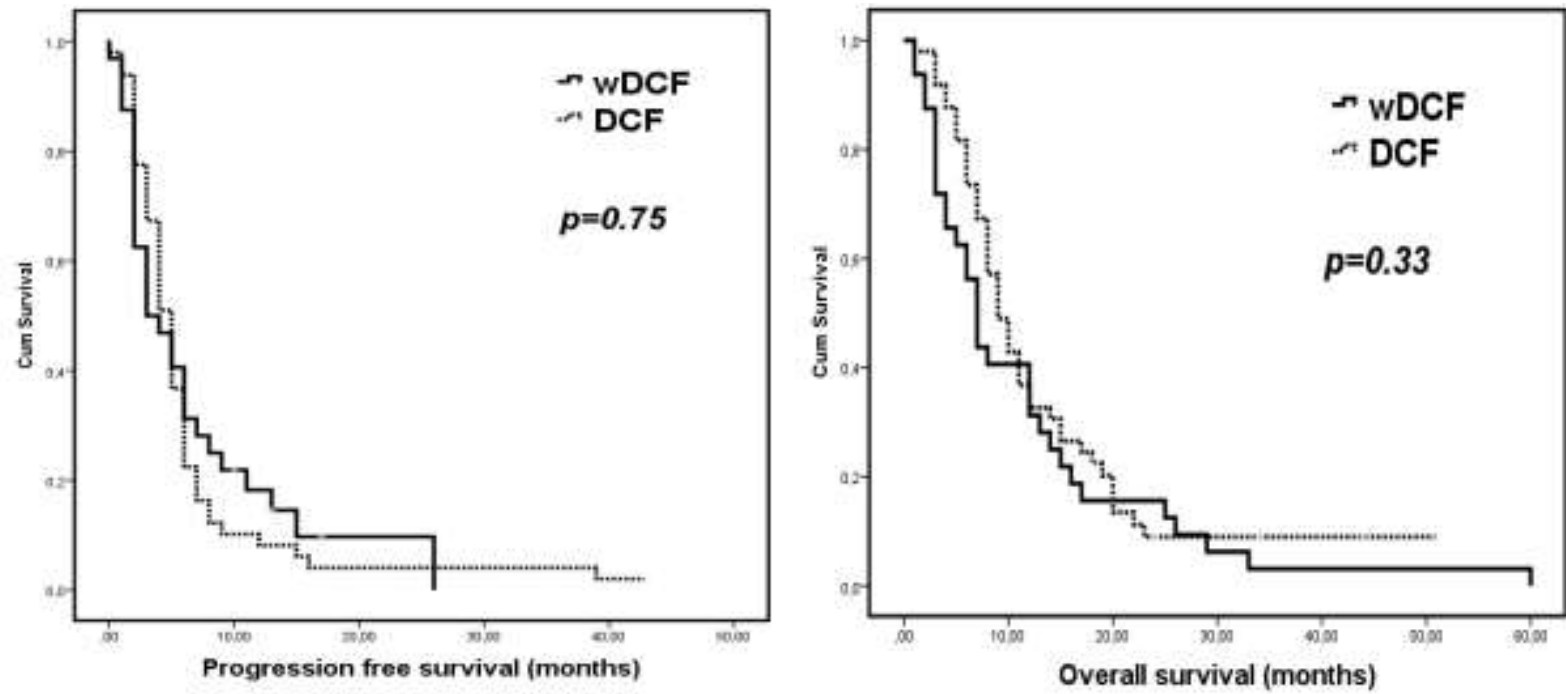

Figure: Progression free survival and overall survival. 